\title{
Physiologische Konzepte von Galen bis Haller
}

\author{
Von Nikolaus Mani
}

Das Wort Physiologia bezeichnete bis weit in die Neuzeit ganz allgemein Naturlehre und Naturphilosophie. Physiologie im modernen Sinne wurde in der Antike und im Mittelalter als die Lehre von den natürlichen Verrichtungen des Körpers «actiones naturales corporis» (Celsus) bezeichnet, vor allem aber nach Galen mit dem Ausdruck usus, utilitas partium, Chréia moríon belegt, d.h. der Wissenschaft vom funktionellen Nutzen der Körperteile. In der Mitte des 16. Jahrhunderts erhielt der Terminus Physiologie bei Jean Fernel eine speziellere Bedeutung und beschrieb die elementare und stoffliche Zusammensetzung sowie den Bau und die Verrichtung der Körperorgane. Seit der Mitte des 18. Jahrhunderts definierte Haller die Physiologie als bewegte und belebte Anatomie (Anatome animata) und als Lehre der physikalischen und chemischen Prozesse der Körpermaschine.

\section{Physiologie bei Galen}

Der Arzt, biologische Forscher und Philosoph Galen gab im 2. nachchristlichen Jahrhundert eine umfassende Darstellung der allgemeinen und speziellen Physiologie und beschrieb mit einer für die Antike seltenen Präzision die anatomisch-präparative und physiologisch-experimentelle Technik ${ }^{1}$. Die galenische Physiologie wurzelt im gesamten antiken Erdreich. Sie wird geprägt durch die platonische Naturphilosophie, die aristotelische Biologie, die alexandrinische Anatomie und physiologische Experimentation ${ }^{2}$. Der Organismus, so führt Galen aus, ist vom Schöpfer planvoll aufgebaut. Er entsteht nicht aus einer zufälligen Aggregation von ungeordnet im leeren Raum durcheinanderwirbelnder Atome. Diese Vorstellung der epikureischen Atomisten weist Galen entschieden zurück ${ }^{3}$.

Die Physiologie Galens ruht auf drei Pfeilern: 1. Ein naturphilosophischer Überbau, der teleologisch-vitalistisch orientiert ist. 2. Die Kenntnis der Topographie und Struktur der Organe, die über ihre Verrichtungen wichtige Aufschlüsse erteilt. 3. Als Drittes kommt die unmittelbare physiologische Beobachtung und das Tierexperiment: die Frage nach dem Wie vitaler Phänomene. 
Drei Zentralorgane mit ihren ausführenden Kanälen beherrschen die Lebensprozesse. Die Leber ist Werkzeug der Blutbildung und Wurzelstock der Venen. Über die Venen strömt das in der Leber erzeugte Nährblut in den gesamten Körper ${ }^{4}$. Das linke Herz ist die Quelle der Lebenskraft und Zentralorgan der Arterien. Die linke Herzkammer ist auch Herd der Lebenswärme. Das dickflüssige hepatische Nährblut fließt ins rechte Herz und wird durch feine Poren des Herzseptums in die linke Herzkammer filtriert. Dort vermischt sich das Blut mit der Atemluft und wird durch die Hitze des linken Ventrikels in ein warmes, hellrotes Blut umgewandelt, das mit dem Lebenshauch (Pnéuma zotikón, spiritus vitalis) begabt ist und das über den Herz- und Arterienpuls in den gesamten Körper ausströmt und alle Organe vitalisiert ${ }^{5}$. Das dritte Kardinalorgan ist das Gehirn. Es ist Zentralorgan der Nerven und Ursprung von Bewegung, Empfindung und geistiger Tätigkeit, d.h. Vorstellungskraft (Phantasía), Verstand (Epistéme, Dianóesis), Gedächtnis (Mnéme) und Erinnerungsvermögen (Anamnesis) ${ }^{6}$. Ein feinstofflicher Seelenhauch (Pnéuma psychikón) wird aus dem arteriellen Blut und aus der Atemluft in die Hirnhöhlen abfiltriert und strömt über die Nervenkanäle in den Körper, wobei es Empfindung (Aísthesis) und Bewegung (Kínesis) vermittelt ${ }^{7}$.

Für die Antike ungewöhnlich ist Galens Definition eines Organes und die Verknüpfung von Bau und Tätigkeit der lebenden Gebilde. Unter einem Organ versteht Galen einen Körperteil, der sich aus verschiedenen homogenen Bestandteilen, den Homoiomeren (den Geweben), zusammensetzt ${ }^{8}$. Dabei ist die Anordnung dieser homogenen Bestandteile von höchster Zweckmäßigkeit für die Tätigkeit des Organs. Galen fragt zunächst: Wozu ist ein Organ da, worin besteht sein Nutzen für den Gesamtkörper? Dann aber sucht er auch die Frage zu beantworten, wie das Organ arbeitet.

Ein Beispiel: Die Leber ${ }^{9}$. Sie ist Werkzeug (Organon) der Blutbildung und Ernährung und setzt sich aus dem Leberfleisch oder dem Parenchym, aus Blutgefäßen, Gallenkanälen und einem peritonealen Überzug zusammen. Spezifischer Träger der Leberfunktion ist das Parenchym. Es ist warm, rötlich und geronnenem Blute ähnlich und vermag den über die Pfortader zugeführten Nahrungsbrei zu assimilieren (Homoióo, Homóiosis), d.h. sich gleich zu machen. Dieser Assimilationsprozeß geschieht durch eine spezifische in der Physis des Parenchyms wurzelnde Vitalkraft. Produkt der Leberassimilation ist das hepatische Nährblut, das dem Leberfleisch ähnlich und daher auch blutartig ist. Die blutbildende Assimilation bedarf der Hilfsinstrumente: 1. Einmal die Lebervenen, die das frisch erzeugte näh- 
rende Leberblut über die Zweige der unteren und oberen Hohlvene in den ganzen Körper verteilen. 2. Im Verlaufe der Blutbildung entstehen Exkretionsstoffe. Einmal die gelbe, heiße Galle, die über die Gallenwege in den Darm abfliesst, diesen zur Bewegung stimuliert und den Kot braun färbt. Dann die erdig-kalte schwarze Galle, die über die Vena lienalis in die Milz angezogen wird. Der Überschuß an Blutwasser schließlich sickert durch eine spezifische vitale Attraktion aus den Nierenvenen ins Nierenparenchym und wird daraus als Urin über die Harnwege abgeleitet.

Wie sinnvoll der anatomische Bau auf die funktionellen Bedürfnisse abgestimmt ist, zeigt die Verzweigung der Pfortader in kleinste Blutgefäße, die das Leberfleisch engmaschig durchsetzen. Dadurch entsteht ein breitflächiger Kontakt für die blutbildende Einwirkung der Lebersubstanz auf den fein verteilten Nährbrei. Die Tätigkeit und das Zusammenspiel der Organe zum Wohle des Organismus enthüllt die vollkommene Planung des Schöpfers.

Für die Antike selten und ungewöhnlich sind Galens Tierversuche. Galen durchtrennt am lebenden Tier das Rückenmark auf verschiedener Höhe ${ }^{10}$. Die unterhalb der Schnittstelle abgehenden Nerven waren gelähmt, und die von ihnen versorgten Körperregionen waren der Empfindung und Bewegung unfähig. Mit aufsteigender Durchtrennung des Rückenmarks wurden zuerst die unteren Gliedmassen, dann die Muskeln des Rumpfes und Brustkorbs gelähmt. Nach querer Durchschneidung des oberen Halsmarks erstickte das Tier, weil alle Atemmuskeln mit Einschluß des Zwerchfells gelähmt waren ${ }^{11}$. Ligatur oder Sektion der Nn. recurrentes (Neúra palindromúnta) lähmte die Kehlkopfmuskeln und führte zu Heiserkeit oder Stimmlosigkeit. Galen nannte die Nn. recurrentes daher auch Stimmnerven (Neúra phonetiká) ${ }^{12}$.

Nach Inzision einer Arterie bricht ein pulsierender Blutstrahl hervor, der den Blutgehalt der Arterien anzeigt. Die Arterien enthalten vornehmlich warmes, hellrotes und dünnflüssiges Blut und nicht allein Luft, Spiritus vitalis oder Äther in reiner Form ${ }^{13}$.

Werden die Harnleiter unterbrochen, schwellen sie nierenwärts an und zeigen damit die Richtung des Urinflusses ${ }^{14}$. Allerdings: Das physiologische Experiment erhärtet und bestätigt das geschlossene anatomisch-physiologische und naturphilosophische System Galens; das Experiment ist aber nicht alleinige Quelle und Prüfstein physiologischer Erkenntnis.

Galen ist überzeugt, daß erst die Kenntnis von Bau und Leistung der Organe - also Physiologie im weiteren Sinne - eine wissenschafliche Medizin 
begründen kann. Der Arzt, der eine wissenschaftliche Methode und Therapie (logikè méthodos, logikè therapéia) anwendet, berücksichtigt drei Ebenen theoretischer Überlegungen ${ }^{15}$. Er beachtet die Physis der primären Elemente (Próta stoichéia) und die Mischung der Elemente und Qualitäten, also die stofflich-dynamischen Eigenschaften ${ }^{16}$. Der Arzt muß ferner mit der Natur der sekundären, sichtbaren Elemente, d.h. der homoiomeren oder einfachen Körperteile (Haplà mória) vertraut sein ${ }^{17}$. Schließlich muß der wissenschaftlich denkende und handelnde Arzt auch die Struktur (Diáplasis, Kataskeué organiká) der zusammengesetzten Körperteile, d.h. der Organe (Mória organiká) kennen ${ }^{18}$, er muß über die Lage (Thésis) und Leistung (Enérgeia) der Organe Bescheid wissen und die funktionelle Dignität (Kyriótes) derselben berücksichtigen ${ }^{19}$. Der Chirurg muß ebenfalls über anatomisch-topographische und funktionelle Kenntnisse verfügen. Er soll z. B. bei Entfernung von gebrochenen Schädelknochen einen Druck auf das Gehirn vermeiden, weil der Patient sonst bewußtlos wird oder stirbt ${ }^{20}$. Bei Halsoperationen muß man sich vor einer Verletzung der Nn. recurrentes hüten, da sonst Heiserkeit oder Aphonie entsteht ${ }^{21}$. Wenn in der Brustbeingegend operiert wird, dürfen die Aa. thoracicae internae nicht durchschnitten werden, um schwere Blutungen zu vermeiden ${ }^{22}$. Vor allem darf man bei Brustoperationen das Brustfell nicht durchbohren (Sýntresis), da sich in diesem Falle der Brustkorb mit Luft füllt und die Lungen von außen zusammengedrückt werden ${ }^{23}$.

\section{Das 16. Jahrhundert: Anfänge empirischer Physiologie}

Zu Ende des 15. und im Laufe des 16. Jahrhunderts erhielt die zum großen Teil an Galen anknüpfende medizinisch-biologische Grundlagenforschung neue Impulse. Die pathologischen Sektionen, die Antonio Benivieni ${ }^{24}$ durchführte, die Untersuchungen über den Bau und die Funktionen des menschlichen und tierischen Organismus durch Leonardo da Vinci ${ }^{25}$, die Begründung einer exakten menschlichen Anatomie durch Vesal ${ }^{26}$, die embryologischen Forschungen von Fabricius ab Aquapendente ${ }^{27}$ und die vergleichend-anatomischen Studien Volcher Coiters ${ }^{28}$ bezeugen eine kraftvolle empirische Forschung dieser Epoche.

Aber neben diesen fundamentalen morphologischen Untersuchungen gibt es in dieser Zeit auch Ansätze zu einer experimentellen Physiologie, die bis ins Detail auf Galen zurückgeht ${ }^{29}$. Im Gegensatz zu Galen wird nun aber 
dem experimentellen Befund ein höherer Erkenntniswert beigemessen. Grundlage der physiologischen Methode bildet die Vivisektion (sectio vivorum; viva sectio) ${ }^{30}$. Die anatomische Beobachtung an der Leiche - so stellen einige Forscher fest - vermag für sich allein die Arbeitsweise eines Organs nicht zu klären.

Dazu bedarf es auch der experimentellen Analyse am lebenden Tier. Vesal stellt im vivisektorischen Appendix seiner «Fabrica» fest: «Ita quoque vivi animantis sectio interim functionem ipsam manifesto ostendit.» ${ }^{31}$ (So zeigt auch die Sektion am lebenden Tier manchmal die Funktion selbst deutlich auf.) Realdo Colombo äußert sich noch bestimmter: Die Tätigkeit der Organe können wir nur durch Versuche am lebenden Tier erkennen (Actionem scire nullo pacto possumus, nisi viva sectione utamur) ${ }^{32}$. Um die Funktion der Brustorgane zu beobachten, führte Vesal die künstliche Beatmung des Versuchstieres ein; dadurch wurde der tödliche Pneumothorax vermieden. Vesal eröffnete die Trachea, führte ein Röhrchen in dieselbe ein und blies damit die Lungen auf. Die kollabierten Lungen erweiterten sich, und das matt schlagende Herz erwachte zu kräftigem Pulsschlag ${ }^{33}$.

Realdo Colombo untersuchte am lebenden thorakotomierten Versuchstier, dessen Herz und große Gefäße freigelegt waren, folgende Frage: Enthalten die Lungenvenen Ruß, Luft oder Blut? Er fand sie im Gegensatz zur traditionellen Doktrin vornehmlich mit Blut erfüllt und folgerte: Das Blut wird durch die Lungenarterie (Vena arterialis) in die Lungen getrieben, vermischt sich dort mit der eingeatmeten Luft und fließt dann über die Lungenvenen (Arteriae venales) ins linke $\mathrm{Herz}^{34}$. Dies bedeutete nichts mehr und nichts weniger als die experimentelle Demonstration des kleinen Kreislaufs.

\section{Das 17. Jahrhundert: Physiologie im Zeitalter der naturwissenschaftlichen Revolution}

Im Laufe des 17. Jahrhunderts wurde die theoretische Medizin, die bis dahin fest in der aristotelisch-galenischen Überlieferung wurzelte, radikal erschüttert und verändert ${ }^{35}$. Neben die traditionelle auf Antike und Scholastik basierende Physiologie treten neue Denkrichtungen, Konzepte und Methoden zur Deutung und Erklärung vitaler Phänomene. Diese Strömungen sind: 1. Eine empirisch und experimentell orientierte Physiologie. 2. Eine mechanistisch-physikalische Erklärung der Lebenserscheinungen. 3. Eine iatrochemisch inspirierte Physiologie. 4. Eine vitalistisch-hylozoistische 
Interpretation der vitalen Vorgänge und eine «animistische» Reaktion auf die starre rein mechanistische Erklärung der Lebensprozesse.

\section{Empirische Physiologie}

Die wichtigste und unmittelbare erfolgreichste Richtung war die empirisch orientierte Physiologie. Ihre Methoden waren die morphologische Untersuchung der organischen Strukturen und die experimentelle Analyse vitaler Prozesse. Dieses Konzept gründete auf der aristotelischen und galenischen Biologie und setzte die experimentellen Studien Galens und der Renaissance-Anatomen fort. Daneben hatte sie Querverbindungen zu den neuen iatrochemischen und iatrophysikalischen Richtungen, die von der naturwissenschaftlichen Revolution des 17. Jahrhunderts geprägt waren. Der Unterschied zur traditionellen Schule besteht in folgendem: Die anatomische und mikroskopische Beobachtung und die Ergebnisse des Tierversuchs sind nun Quelle und Prüfstein physiologischer Erkenntnis. Die Forderung nach einer auf Erfahrung gründenden Wissenschaft ertönt allenthalben und erfaßt Philosophie, Physik, Physiologie und Heilkunde. Francis Bacon, Lordkanzler und Philosoph, Vertreter der induktiven Methode, entwarf in seiner utopischen Schrift «Nova Atlantis» ${ }^{36}$ eine prophetische Vision empirischer Naturforschung: Geologische Stationen unter der Erde, astronomische und meteorologische Observatorien auf Türmen und Bergspitzen, physiologische und pharmakologische Laboratorien, wo Gifte und Heilmittel geprüft und neue Tierarten erzeugt werden. Im Jahre 1600 erschien die klassische Untersuchung William Gilberts über den Magnetismus ${ }^{37}$, und 1628 forderte und verwendete William Harvey in seinem epochalen Werk über die Bewegung des Herzens und des Blutes folgende Methoden ${ }^{38}$ : Eigene Beobachtung (autopsia; experimenta ocularia), Tierversuche (dissectio vivorum; experimenta vivorum) und quantitativ-numerische Registrierung physiologischer Prozesse (calculus; computatio). Niels Stensen unterstreicht die Notwendigkeit unablässiger Beobachtungen (necessitas observationum), die allein zu neuen Entdeckungen führen ${ }^{39}$. Th. Bartholin stellte lapidar fest: Wir glauben nur, was wir mit den Augen sehen und mit Händen greifen können (nos tantum credimus, quantum manibus palpamus oculisque videmus) ${ }^{40}$. Johann Jacob Wepfer in Schaffhausen vertraut allein Auge und Hand, um die Evidenz sichtbarer und greifbarer anatomischer Gebilde zu demonstrieren ${ }^{41}$. Wepfers Schüler Johann Conrad Brunner stellt fest: 
Ärztliche Erkenntnis beruht auf Erfahrung (experientia), d.h. auf dem Sammeln und Auswerten zahlreicher gleichartiger Beobachtungen (sylloge et observatio eius, quod saepius et eodem modo visum est) ${ }^{42}$.

William Harveys Entdeckung des Blutkreislaufs verkörpert die neuen Konzepte und Methoden: 1. Die «Autopsia» zeigt am lebenden Tier eine beträchtliche Entleerung der Herzkammern in der Systole. 2. die vergleichende Physiologie der Wirbellosen, Fische, Schlangen, Säugetiere demonstriert einen einheitlichen Funktionsplan. Die Venen führen Blut zum Herzen, die Arterien empfangen das vom Herzen ausgetriebene Blut. 3. Die Anordnung der Venenklappen beweist die herzwärts gerichtete Strömung des venösen Blutes. 4. Die Unterbindung der Aorta und Vena cava im vivisektorischen Experiment zeigt den peripheren Blutfluß in den Arterien und die herzwärts gerichtete Strömung in den Venen. 5. Aus der quantitativen und numerischen Betrachtung (Calculus) ergibt sich folgendes. Der linke Ventrikel faßt etwa zwei Unzen (ca. $60 \mathrm{ml})$. Wegen der beträchtlichen systolischen Verkleinerung des Ventrikels kann man das Schlagvolumen auf eine Unze $(30 \mathrm{ml})$, eine halbe Unze oder noch weniger schätzen. In einer halben Stunde zu 1000 Pulsschlägen treibt das Herz bei einem Schlagvolumen von 1 Unze etwa 1000 Unzen oder gut 83 Pfund Blut in die Peripherie und empfängt die gleiche Blutmenge aus der Vena cava. Woher kommt diese Blutmenge, und wohin fließt sie ab? Der Blutersatz aus der Nahrung vermag keine 83 Pfund pro halbe Stunde zu ersetzen. Also muß sich das Blut im Kreise bewegen. Die Kreisbewegung aber ist für den Aristoteliker Harvey eine im kosmischen (Planeten) und im terrestrischen Bereich (Wasserkreislauf) ideale Bewegung. Der motus circularis geschieht auch im Mikrokosmos Mensch, dessen Sonne im Herzen liegt. Das Sonnenherz strahlt Lebenskraft aus und erquickt und belebt das verbrauchte Venenblut zu neuer Vitalität.

Einige weitere Beispiele sollen diese empirisch und experimentell orientierte Physiologie illustrieren. Die morphologische Beschreibung der physiologischen Apparate stützt sich auf anatomische Zergliederung, Gefäßinjektionen und mikroskopische Untersuchungen (Anatomia subtilis et artificiosa). Francis Glisson ${ }^{43}$ demonstriert die Blutversorgung der Leber mit einem zweiten, aus der Pfortader entspringenden Kapillarnetz, das sich im Innern der Leber aufteilt. Niels Stensen und Marcello Malpighi beschreiben die Struktur der Drüsen mit der zuführenden Arterie, der abführenden Vene und dem exkretorischen Kanal, der den Drüsensaft ableitet ${ }^{44}$. Malpighi charakterisiert das nach ihm benannte Nierenkörperchen als funktionelle Einheit der Harnabsonderung ${ }^{45}$ und entdeckt die zwischen Arterien und 
Venen eingeschalteten Kapillaren ${ }^{46}$. Antoni van Leeuwenhoek entdeckt mit den von ihm selbst geschliffenen Linsen die roten Blutkörperchen, beobachtet die kapillare Zirkulation in vivo, beschreibt fettartige Chyluskörperchen und die gestreiften Fasern des Herzmuskels ${ }^{47}$. Die Unterbindung von Blutund Lymphgefäßen zeigt die Richtung der Saftströme. Die partielle oder totale Exzision verschiedener Organe entscheidet die Frage nach ihrer Lebensnotwendigkeit. Milzlose Tiere z. B. überleben ${ }^{48}$. Johann Conrad Brunner ${ }^{49}$ entfernt bei Hunden den größten Teil des Pankreas, wobei einige Tiere den Eingriff überleben. Die Versuchsprotokolle vermerken, daß die Tiere hungrig und durstig werden und viel Harn lassen. Reinier de Graaf ${ }^{50}$ erweiterte das Feld der operativen Physiologie und legte bei Hunden Speichel-, Pankreas- und Gallenfisteln an, um die Drüsensäfte chemisch zu untersuchen. Drogen und Gifte wurden per os verabreicht und seltener intravenös eingespritzt, um das pharmazeutische Drama in der Tiefe des Organismus zu verfolgen (Thomas Willis) ${ }^{51}$. Richard Lower ${ }^{52}$ eröffnete am künstlich beatmeten Tier die Brusthöhle und zeigte, wie sich das dunkle, venöse Blut bei der Lungenpassage purpurrot färbt. Jan Swammerdam ${ }^{53}$ untersuchte am isolierten neuromuskulären Präparat des Frosches die Muskelkontraktion und wies nach, daß der Muskel auch ohne cerebrale Impulse durch Reizung seiner Nerven zum Zucken gebracht wird.

Die Versuchsbedingungen wurden mit einer bis dahin unbekannten Sorgfalt registriert und reproduzierbar gemacht: Art, Geschlecht, Alter, Gesundheits- und Ernährungszustand der Versuchstiere wurden angegeben. Die experimentelle Technik wurde im Detail beschrieben, der Ablauf der Experimente protokolliert, und manchmal wurde auch eine autoptische Kontrolle der Versuchsanordnung vorgenommen.

\section{Mechanistisch-physikalische Erklärung der Lebenserscheinungen}

Zahlreiche Philosophen und Naturforscher des 17. Jahrhunderts führen alle Phänomene der toten und belebten Natur auf die Wirkungen bewegter Materie zurück. Mechanistisch-physikalische Vorstellungen und Modelle wurden entwickelt, um die vitalen Prozesse zu erklären. Hier erfaßte die naturwissenschaftliche Revolution intensiv die Gebiete der Physiologie und theoretischen Medizin. Pierre Gassendi ${ }^{54}$ entwickelt auf der Grundlage des antiken Atomismus eine korpuskuläre Physik. Die anorganischen und organischen Phänomene resultieren aus der Anordnung und Bewegung kleinster Teilchen im leeren Raum. Die größeren und dichter angeordneten 
Partikel bilden Knochen und Fleisch. Subtile, dynamische Teilchen durchströmen die festen Organe und versetzen sie in vitale Aktion. Die Anima sensitiva der Alten wird nun durch rein physikalisch-materielle Mechanismen ersetzt. Descartes ${ }^{55}$ betrachtet den menschlichen und tierischen Leib als Maschine. Alle Funktionen des Körpers resultieren aus der Anordung der organischen Strukturen, die ganz und gar physikalischer Gesetzlichkeit unterworfen sind. Die den cartesischen Automaten antreibende Kraft ist ein im Herzen brennendes Feuer. Diese Lebensflamme ist von gleicher Qualität wie das gewöhnliche Feuer, sie dehnt das Blut aus und treibt es im Kreise herum. Niels Stensen fordert für die Beschreibung vitaler Phänomene mathematische Genauigkeit und erklärt die Muskelkontraktion als geometrische Verschiebung der kontraktilen Fasern und preist Gott als sublimen Mechaniker ${ }^{56}$. In der Naturlehre (Physica) hat nur jenes Wissen Bestand, das wir durch Erfahrung und Beobachtung gewonnen und durch die naturwissenschaftliche Theorie physikalisch-mechanisch abgeleitet haben: «In physica nihil scimus praeter experimenta et observationes eaque, quae ex ipsis iuxta principia metaphysica et mechanica deducuntur.» ${ }^{57}$ Giovanni Alfonso Borelli, Arzt und Physiker aus der Schule Galileis, charakterisiert die vitalen Phänomene als mechanische Vorgänge, die mathematisch-geometrisch beschrieben werden müssen. Das Buch der Natur, so verkündet er, ist von Gott in mathematischen Ziffern geschrieben ${ }^{58}$. Das posthum erschienene, mit prachtvollen und präzisen Illustrationen versehene entomologisch-biologische Werk Jan Swammerdams betitelt sich «Bibel der Natur», natürliche Offenbarung gesellt sich zu religiöser Erleuchtung. Borelli entwirft physikalische Modelle zur Darstellung physiologischer Vorgänge, etwa der Muskelkontraktion oder der venösen Klappenfunktion ${ }^{59}$. Physiologie wird bei ihm zur Physik: Das Herz arbeitet als Hohlmuskel, für die Leistungen der äußeren Muskelarbeit sind die Hebelgesetze gültig, und die Drüsen arbeiten als mechanische Filter. Borelli erklärt sich z. B. die Muskelkontraktion so. Durch eine explosive Fermentation, die dem Aufbrausen einer Mischung von Säure und Base gleicht, werden die Porositäten im Innern des Muskels erweitert. Der Muskel bläht sich dabei auf und wird hart ${ }^{59}$ a Eine solche «operatio mechanica» ist die einfachste und überzeugenste Erklärung für die Muskelkontraktion und entspricht schon deshalb der Wirklichkeit, weil die Natur schon zum vornherein einen modus operandi hat, der physikalisch einfach und leicht zu bewerkstelligen ist ${ }^{59 \mathrm{~b}}$ : «Praeterea, quod nedum possibilis sit, sed etiam necessario admittenda sit talis mechanica operatio, suademur ex eo, quod exacte phaenomenis satis 
facit; et quia nullus alius modus possibilis et facilior occurrit, et quia Natura nunquam consuetos, faciles et obvios operandi modos relinquit.» Johann I. Bernoulli bedient sich der Infinitesimalrechnung, um die Druckverhältnisse im efferveszierenden Muskelbläschen zu berechnen, wobei er dieses Bläschen als physiko-chemisch arbeitende Funktionseinheit des Muskels betrachtet $^{59 \mathrm{c}}$.

Stensen postulierte 1665 folgendes Axiom für die medizinische Forschung ${ }^{60}$. Der Körper arbeitet als Maschine. Krankheit ist Folge eines Maschinendefektes. Therapie bedeutet Reparatur des Maschinenschadens. Um aber eine Maschine zu kennen, muß man sie in ihre Bestandteile zerlegen. Bei der organischen Maschine bedeutet dies: anatomische Zergliederung, Studium der embryologischen Entfaltung, experimentelle Untersuchung der funktionellen Leistungen.

Malpighi äußerste sich zu dieser Frage folgendermaßen ${ }^{61}$. Die Natur arbeitet streng gesetzmäßig und uniform «La natura opera per necessità sempre uniforme» ${ }^{62}$. Deshalb wird es dem Menschen auch gelingen, einen beträchtlichen Teil der kunstvollen Einrichtungen zu enthüllen, z.B. die Hebelwirkung der Gelenke, die Art der Strömung von Blut und Lymphe, den Mechanismus der Drüsenfilter, die chemische Zusammensetzung des Blutes ${ }^{63}$. Dabei spielen sich diese physiko-chemischen Prozesse an Mikroapparaten ab, z.B. den Nierenkörperchen. Mikroskopische Beobachtungen entspringen dem Bedürfnis, Struktur und Tätigkeit dieser winzigen Maschinen zu erforschen, die aus Hebeln, Bälkchen, Fäserchen, Filtern u.ä. bestehen.

Die letzte Ursache des Lebens, so unterstreicht Malpighi, bleibt verborgen, aber Struktur und mechanische Funktion der tierischen und pflanzlichen Apparate ist prinzipiell erforschbar. Wie die Seele sich des Körpers bedient, wissen wir nicht, aber es ist gewiss, daß sie auf eine Maschine einwirkt und diese in Bewegung setzt. Eine gegebene Maschine arbeitet immer auf dieselbe gesetzmässig determinierte Weise, gleichgültig, ob sie von Mensch, Pferd, Wasserkraft oder gar von einem Engel angetrieben wird $^{64}$. Robert Hooke vermutete in seiner berühmten «Micrographia», daß nicht okkulte Qualitäten oder Kräfte, sondern daß die Mikrotextur der winzigen Maschinen der Natur die physiologischen Funktionen bedinge ${ }^{64 \text { a }}$.

Auch die quantitative Beschreibung vitaler Vorgänge dringt Hand in Hand mit der physikalischen Denkweise in die Physiologie ein. Santorio Santorio versuchte zu Beginn des 17. Jahrhunderts in seiner «Medicina statica» ${ }^{65}$ den Stoffumsatz an Tausenden von Versuchspersonen, darunter 
auch Galilei, messend zu bestimmen ${ }^{66}$. Mit Hilfe der Waage bestimmte er den Gewichtszuwachs durch Speise und Trank und den Gewichtsverlust durch Kot und Urin. Dabei zeigte sich, daß bei gleichbleibendem Gewicht ein beträchtlicher Teil der aufgenommenen Nahrung den Körper als unsichtbare Ausdünstung (perspiratio insensibilis) über Haut und Lungen verlassen mußte. Von 8 Pfund Speise und Trank, die eingenommen wurden, betrug die unsichtbare Ausdünstung ca. 5 Pfund ${ }^{67}$. Weitere Beispiele quantitativer Untersuchungen sind die Bestimmung der muskulären Hubkraft durch Borelli, die Messung der Sekretionsgröße von Leber und Pankreas durch de Graaf, dann die Schätzung des Herzschlagvolumens durch Harvey.

\section{Iatrochemisch inspirierte Physiologie}

Die von Paracelsus und van Helmont inspirierte Schule der Chemiatrie versuchte, die Phänomene von Gesundheit und Krankheit als chemische Prozesse zu deuten. Van Helmont glaubte, daß die Stoffumwandlung im Organismus durch «Fermente» gesteuert werde. Ein Ferment, so führt van Helmont aus, besteht aus einem materiellen Träger und einem geistigen vital-dynamischen Prinzip, einem Arcanum vitale ${ }^{68}$. Franciscus de le Boe Sylvius erklärte die Entstehung der Körperwärme als chemische Reaktion: Saurer Chylus und alkalische Galle vereinen sich im Herzen unter Aufbrausen und Wärmeentwicklung ${ }^{69}$. Die Polarität zwischen Säuren und Basen ersetzt hier die frühere Säfte- und Qualitätenlehre.

\section{Eine vitalistisch-hylozoistische Interpretation}

Neben physiko-chemischen und mechanistischen Deutungen der Lebensprozesse werden auch vitalistische Konzepte entwickelt. Francis Glisson ${ }^{70}$ glaubt, daß Gott die Materie selbst mit vitalem Reaktionsvermögen ausgestattet habe. Diese vitale Grundeigenschaft potenziere sich auf der organischen Stufenleiter bis zur bewußten Empfindung und Beantwortung von Reizen. Alle Gewebe des Organismus sind mit der Fähigkeit der Perzeption (perceptio), des Verlangens (appetitus) und der Bewegung (motus) versehen. Aus dieser Trias erklärt sich die Irritabilität, d.h. die unbewußte automatisch-gesetzmäßige und die bewußte Reaktion lebender Substanz auf innere und äußere Reize. Georg Ernst Stahl ${ }^{71}$ vertritt ein neues animistisch-vitalistisches Konzept des «Organismus». Gewiß gibt es, so sagt Stahl, eine 
instrumentale mechanische Anordnung der animalen Maschine, wo die physikalischen Gesetze gültig sind. Aber dies allein genügt nicht zu einer befriedigenden Erklärung vitaler Phänomene. Der «Organismus» ist mehr als nur «Mechanismus» (De differentia mechanismi et organismi) ${ }^{72}$. Der Leib wird durch die «anima» regiert. Anima ist Lebensprinzip (principium vitale, Physis, ens vitale etc.). Und dieses Prinzip vermittelt Lebenskraft (robur vitale, energia vitalis). Die anima wirkt dem spontanen Zerfall organischer Materie durch einen motus conservatorius entgegen, sie garantiert eine Harmonie der Funktionen (harmonia functionum), das Zusammenspiel der Organe (Synergia partium). Kurz, die organische Tätigkeit ist zweck- und zielgerichtet, eine directio organica mit einer intentio finalis.

In der zweiten Hälfte des 17. Jahrhunderts werden Ärzte zu Naturforschern und umgekehrt widmen sich viele Naturwissenschaftler medizinischen Problemen. Borelli ist Physiker und Mediziner, für den Leben Biomechanik und Chemie bedeutet. Stensen ist Physiologe, Anatom, Embryologe, Paläontologe, bevor er Theologe und Priester wird. Der Physiker Robert Hooke und der Chemiker Robert Boyle unternehmen physiologische Experimente, führen die Technik der künstlichen Beatmung ein, untersuchen den Einfluß des Vakuums auf Versuchstiere und vergleichen die Veränderung der Luft durch das atmende Tier und die brennende Kerze.

Die Forschung verlagert sich zu einem großen Teil extra muros universitatis in private Zirkel und Schulen, z.B. die nicht institutionalisierte Schaffhauser Ärzteschule unter Wepfer, vor allem aber in die neugegründeten Akademien ${ }^{73}$. Hier wurden neue Apparate ersonnen und vorgeführt, z. B. das Barometer, das Thermometer, die Luftpumpe, das Mikroskop. In den Akademien treffen sich Ärzte und Naturforscher, überhaupt die curiosi naturae verschiedener Herkunft, zur Diskussion und Demonstration neuer Entdeckungen und Verfahren. Hier befaßt man sich mit dem Einfluß des Luftdrucks auf den Organismus, hier wird die innere Atmung untersucht, das Problem der Urzeugung erörtert und experimentell überprüft. Antoni van Leeuwenhoek, Tuchhändler, Eichmeister und Vermessungsbeamter der Stadt Delft, teilt seine erstaunlichen mikroskopischen Beobachtungen der Royal Society zu London mit, in deren Transactions sie publiziert werden und weiteste Verbreitung finden.

Die Frage nach dem Wesen und den Methoden medizinischer und naturwissenschaftlicher Forschung wird intensiv erörtert. Stensen und Malpighi fordern ein der Forschung günstiges Klima: wissenschaftliche 
Freiheit, Gemütsruhe, Muße und Zeit (libertà, filosofia libera, ozio) ${ }^{74}$. Innovation und Akzeleration der zeitgenössischen Forschung wird lebhaft empfunden ${ }^{75}$. Die neuen Entdeckungen, Kenntnisse und Konzepte werden nicht allein als Zuwachs und Ergänzung traditionellen Wissens betrachtet, man deutet sie vielmehr als qualitativen Bruch mit der Überlieferung und führt sie auf das neue naturwissenschaftliche Denken zurück. Die moderne Physiologie und Medizin gelten vielfach als Teilgebiete der Naturwissenschaften. Neben enthusiastischer Zustimmung sind aber auch warnende Stimmen zu hören. Jean Riolan ${ }^{76}$ als Verteidiger der galenistischen Bastion warnt vor der Hybris der Forscher, die auch vor Versuchen am Menschen nicht zurückschrecken würden. Jan Swammerdam weist auf die Grenzen naturwissenschaftlicher Erkenntnis hin. Man könne sein Leben lang die Muskelbewegung studieren, um letzten Endes die Schranken menschlicher Einsicht zu erkennen. Zudem seien «Wissenschaften und Entdeckungen milde Gaben Gottes, die er nach eigenem Belieben austheilt». ${ }^{77}$ Thomas Sydenham ${ }^{78}$ ist gegenüber der mikroskopischen Forschung skeptisch. Sie überschreite die von Gott gesetzten Sinnesgrenzen und sei wohl von geringem praktischem Nutzen. Das größte Heilmittel des Jahrhunderts, die Chinarinde, welche das Wechselfieber heilt, stamme aus dem südamerikanischen Urwald und habe mit der modernen Forschung nichts zu tun. Medizinisches Wissen, so unterstreicht Sydenham, beruhe auf zwei Hauptdingen: Einmal die präzise ärztliche Beobachtung, aus der ein spezifisches Krankheitsbild herauskristallisiert wird, sodann die Erprobung eines spezifisch wirkenden Heilmittels für die jeweilige spezifische Krankheit. Modell dafür ist die spezifische Wirkung der Chinarinde auf das intermittierende Malariafieber.

Die Physiologie zwischen 1700 und 1760: Physiologie als naturwissenschaftliche Disziplin (St. Hales). Die Synthese der Physiologie als eigenständiges Fach durch Albrecht von Haller

In der ersten Hälfte des 18. Jahrhunderts wird die Newtonsche Physik zum Vorbild nicht nur der exakten Naturwissenschaften, sondern auch der physiologischen Forschung. Ein Beispiel dafür sind Stephen Hales' klassische Untersuchungen über die Hämodynamik ${ }^{79}$. Sie gründen auf John Lockes sensualistischer Philosophie ${ }^{80}$ und auf Newtons beispielhafter experimenteller Analyse der Optik ${ }^{81}$. 
Hales' wissenschaftliches Credo lautet: Nicht reine Spekulation (meer speculations of the mind), sondern zuverlässige experimentelle Daten (proper data of many good and credible experiments), die mathematisch ausgewertet werden ${ }^{82}$, geben erst eine tiefere Einsicht in das wundersame Werk des Schöpfers, in dem alles nach Zahl, Maß und Gewicht wohlgeordnet ist ${ }^{83}$. Zwischen den Dingen, die wir sicher wissen, und der Terra incognita liegt ein Gebiet des Zwielichts. Hier sind Vermutungen (conjectures) erlaubt, die experimentell untersucht, bestätigt oder widerlegt werden und wiederum zu neuen Annahmen führen ${ }^{84}$.

Die tierischen Flüssigkeiten und pflanzlichen Säfte, so unterstreicht Hales, bewegen sich nach hydraulischen und hydrostatischen Gesetzen ${ }^{85}$. Dieser Aspekt der Zirkulation ist aber von den in erster Linie anatomisch orientierten Ärzten vernachlässigt worden ${ }^{86}$. Hales gelang es erstmals, den arteriellen und venösen Blutdruck zu messen ${ }^{87}$. Er führte zu diesem Zweck eine Messingkanüle in das unterbundene Blutgefäß ein und verband die Kanüle mit einem etwa $9 \mathrm{Fuß}$ langen, nach oben führenden Glasrohr gleichen Kalibers. Nun wurde die Ligatur gelöst, und das Blut stieg im Glasrohr an. Die Höhe der Blutsäule gab den Blutdruck (force of the blood) an. Versuchstiere waren Pferde, Hunde und Schafe, am häufigsten wurde der Blutdruck der Schenkelarterien und der Carotis gemessen ${ }^{88}$. Beim Pferd betrug der Blutdruck der Schenkelarterien etwa $8 \mathrm{Fu}$ (ca. $180 \mathrm{~mm} \mathrm{Hg}$ ) der venöse Druck in der Jugularis veriierte je nach dem Erregungszustand des Pferdes zwischen 12 und $48 \mathrm{Zoll} \mathrm{Blutsäule} \mathrm{(ca.} 22-88 \mathrm{~mm} \mathrm{Hg})^{89}$. Weiterhin stellte Hales aufgrund experimenteller Messungen und rechnerischer Überlegungen folgendes fest: 1. Die große Druckdifferenz zwischen dem arteriellen und venösen System ist vor allem durch die arteriellen Kapillaren bedingt, wo der größte Widerstand (resistance) liegt ${ }^{90}$. 2 . Die Herzkraft ist von verschiedenen Faktoren abhängig: von der Ruhe oder Bewegung des Tieres, von der Aufregung, Angst und Schmerz ${ }^{91}$. 3. Beträchtliche Änderungen des Blutdrucks sind mit dem Leben vereinbar; dies beweist eine beträchtliche physiologische Spielbreite (a very considerable latitude in the variation) der wunderbaren vom allweisen Schöpfer erbauten animalen Maschinen ${ }^{92}$. 4. Die systolische Geschwindigkeit des Blutes in der Aorta ergibt sich aus dem Quotienten $\frac{\text { Schlagvolumen/sec } 3}{\text { Querschnitt der Aorta }}$. Der Faktor 3 ist deshalb einzusetzen, weil die systolische Phase etwa ein Drittel der Zeit einer gesamten Herzaktion ausmacht. Beim Hund berechnete Hales eine systolische Geschwindigkeit des Blutes in der Aorta auf ca. 143 Fuß/min (ca. 73 
$\mathrm{cm} / \mathrm{s})^{93}$. Beim Menschen schätzte er das Schlagvolumen auf 1,659 Kubikzoll, während der Querschnitt der Aorta etwa 0,4187 Quadratzoll beträgt. Bei einer Pulsfrequenz von 75 Schlägen pro Minute ergab sich $\frac{75 \cdot 1,659}{0,4187} \quad$ Zoll/min.

Dies entspricht einer Geschwindigkeit der Blutströmung in der Aorta von ca. $74 \mathrm{Fu} 3 / \min (\mathrm{ca} .38 \mathrm{~cm} / \mathrm{s}){ }^{94}$. 5 . Die Arterien werden durch den Impetus des in die Aorta einströmenden Blutes zunächst erweitert, um sich dann nach der Systole zusammenzuziehen. So wird in den kleinen Blutgefäßen (the finer capillaries) eine ausgeglichene Strömungsgeschwindigkeit hergestellt (an almost even tenor of velocity). Hales vergleicht diesen Vorgang mit dem Windkessel der Feuerspritze ${ }^{95}$. 6 . Am lebensfrischen Präparat machte Hales unter physiologischem Druck Perfusionsversuche mit Wasser sowie mit Auszügen von Chinarinde und verschiedener anderer Drogen ${ }^{96}$. Das Volumen der durchströmenden Flüssigkeit variierte je nach der Temperatur des Wassers und der Natur der beigefügten Drogen. Offenbar, so folgerte Hales, gibt es eine Kontraktion (constriction) oder Erschlaffung (relaxation) der Blutgefäße ${ }^{97}$.

Hales' Werk ist charakterisiert durch eine fruchtbare Fragestellung und eine ingeniöse Experimentation, deren klare Befunde mit elementarer Mathematik beschrieben werden und zu grundlegenden Ergebnissen führten. Das Werk «Haemastaticks» gehört zu den klassischen Arbeiten der naturwissenschaftlich arbeitenden experimentellen Physiologie.

Der Mediziner und Physiker Daniel Bernoulli in Basel berechnete um die Mitte des 18. Jahrhunderts erstmals die Herzarbeit (travail du cœur), die er in den richtigen physikalischen Dimensionen als Kraft mal Weg angab, d.h. als Pfund Blut mal Höhe der aufsteigenden Blutsäule aus einer eröffneten Arterie ${ }^{98}$. Dabei stützte er sich in den abschließenden Arbeiten auf die Daten von St. Hales. Bernoulli schätzte das Schlagvolumen des Menschen auf 2 Unzen oder 1/8 Pfund Blut und die Druckhöhe der Blutsäule auf 8 Fuß. Die Herzarbeit des linken Ventrikels beträgt für jeden Pulsschlag $1 / 8$ Pfund Blut mal $8 \mathrm{Fu} \beta=1$ Pfund $11 \mathrm{Fu}$. Schätzt man die Arbeit der rechten Kammer auf den vierten Teil der Arbeit des linken Ventrikels, so verrichtet der Herzmuskel pro Pulsschlag eine Arbeit von 1,25 Pfund Fuß (ca. 0,2 mkg oder ca. 2 Joule). Bernoulli stellte fest: «Man kann die tägliche Herzarbeit auf einen Betrag schätzen, der imstande ist, 144000 Pfund auf $1 \mathrm{Fu} 3$ Höhe zu heben. Dieser Betrag entspricht dem zwölften Teil der täglichen äußeren Arbeit eines Mannes» (1,25 Pfund Fuß mal 115200 Pulsschläge pro 
Tag $=144000$ Pfund $F u ß=24000 \mathrm{mkg}$ ). «On pourra estimer le travail journalier du cœur égal à celui d'élever 144000 livres à la hauteur d'un pied, ce qui fait la douzième partie de ce que l'on peut appeler le travail journalier d'un homme.» ${ }^{98}$ a

\section{Charakterisierung der physiologischen Disziplin durch A. von Haller}

Aufgrund immenser Literaturstudien und systematischer eigener Forschungen schuf Haller eine gewaltige Synthese der Physiologie und charakterisierte erstmals die Physiologie als eigenständige Disziplin ${ }^{99}$. Die Wurzeln von Hallers Physiologie reichen bis in die Antike zurück. Vor allem aber ist sein wissenschaftliches Denken von der anatomisch-physiologischen Forschung zwischen 1540 und 1740 , dem naturwissenschaftlich geprägten Weltbild des 17. Jahrhunderts und vom medizinischen Werk seines Lehrers Hermann Boerhaave bestimmt. Tief beeindruckt ist Haller - wie die meisten seiner Zeitgenossen - von Newton, dem Interpreten terrestrischer und kosmischer Naturgesetze:

«Ein Newton übersteigt das Ziel erschaffener Geister,

findt die Natur im Werk und scheint des Weltbaus Meister...

und schlägt die Tafeln auf der ewigen Gesetze,

die Gott einmal gemacht, daß er sie nie verletze.» ${ }^{100}$

Newtons Forschungsmethode ist für Haller vorbildlich: Man muß die Naturerscheinungen beschreiben und gesetzmäßig verknüpfen, auch wenn man die letzte Ursache nicht kennt: «In hac philosophia propositiones deducuntur ex phaenomenis et redduntur generales per inductionem.» ${ }^{101}$ Haller glaubte, daß man über die Naturkräfte nur das erfahren könne, was die Experimente zeigten, und daß die Wirkung den Gradmesser der Kräfte abgebe, wobei die Natur der Bewegung selbst niemandem bekannt sei: «Nihil enim de viribus novimus, quam quae per experimenta didicimus.» ${ }^{102}$ «Virium mensura effectus est, nam motus ipsius, rei quidem notissimae, naturam tamen nemo philosophorum perspexit.» ${ }^{103}$ Haller umschreibt die Aufgaben der Physiologie folgendermaßen: Die Physiologie untersucht die inneren Bewegungen des Körpers (motus internos), sie erforscht die Verrichtungen der Eingeweide (viscerum munera), sie sucht nach den Gründen, die den Körper am Leben erhalten und das Leben weitergeben, sie beschreibt die Kräfte, die den Muskel bewegen, sie befaßt sich mit der Übermittlung der 
Sinneseindrücke an die erkennende Seele und sie untersucht die Bedingungen der Umwandlung von Nahrung in körpereigene Substanz: «Qui physiologiam scribit, corporis animalis internos motus, viscerumque munera, et humorum mutationes, et vires exponendas sumit, quibus vita sustentatur; quibus rerum species, per sensus acceptae, animae repraesentantur; quibus vicissim lacerti valent, qui mentis reguntur imperio; quibus alimenta in succos nostros, adeo varios, convertuntur; quibus demum ex iis succis, et nostra corpora conservantur, et humani generis iactura novis partibus reparatur. Amplissimum certe pensum, et unius hominis ingenio fere maius.» ${ }^{103 \text { a }}$ Diese Aufgabe ist riesig, so gesteht Haller ein, und übersteigt das Ingenium eines einzelnen Menschen. Schauplatz der Physiologie aber sind die anatomischen Strukturen, deshalb ist Physiologie belebte, bewegte Anatomie: «Physiologia est animata anatomé.» ${ }^{104}$ Die «functiones corporis» sind ohne Kenntnis des Körperbaus nicht zu erfassen. Eine von der Anatomie losgelöste Physiologie wäre ebensowenig leistungsfähig wie eine Mechanik, die die Kräfte einer Maschine mathematisch berechnen wollte, ohne das Räderwerk der Maschine zu kennen ${ }^{105}$. Anatomie bedeutet ästhetischen und intellektuellen Genuß (delectatio intellectualis) und religiöse Erbauung ${ }^{106}$. Die Zergliederung von Mensch und Tier enthüllt den Bau von vollkommenen Maschinen, die von Gott erschaffen wurden. Die Ontogenese zeigt die wunderbare Entfaltung der von Gott im Schöpfungsakt präformierten Keime.

Neben der humanen Anatomie sollte der Physiologe auch die vergleichende Anatomie berücksichtigen. Ein Fehlen der Gallenblase bei gewissen Tieren zeigt, daß dieses Organ nicht lebensnotwendig ist ${ }^{107}$. Die organischen Prozesse sind an kleinste Strukturen gebunden. Nur mit dem Mikroskop erkennt man die Samentierchen und die Form und Bewegung der in den feinsten Gefäßen zirkulierenden Blutkügelchen ${ }^{108}$. Auch die vergleichende Physiologie kann Fragen beantworten: Der Süßwasserpolyp reagiert auf Reize mit Bewegung, ist also irritabel, obwohl er kein Nervensystem besitzt ${ }^{109}$. Auch die pathologische Anatomie trägt zur Kenntnis physiologischer Vorgänge bei: Wenn ein bestimmtes Organ durch Krankheit beschädigt ist, kann man aus dem Ausfall der Funktionen auf die Leistungen dieses Organes schließen. Gedächtnisverlust bei organischen Hirnschäden weist auf eine körperliche Fixierung der Gedächtnisspuren hin ${ }^{110}$.

Wichtigste Methode der Physiologie, die zu unmittelbaren Ergebnissen führt, ist das Experiment am lebenden Tier ${ }^{111}$, die Befragung der Natur unter künstlich variierten Bedingungen. Die Leiche ist bewegungslos. 
Physiologisches Geschehen aber besteht in innerer und äußerer Bewegung des lebenden Organismus. Daher muß am lebenden und sich bewegenden Tier experimentiert werden. Ohne das physiologische Experiment kann weder die Bewegung des Blutes, noch der Lymphstrom, noch die Darmperistaltik, noch die Kontraktion der Muskeln erforscht werden. Ein gültiges Experiment vermag durch lange Tradition sanktionierte Spekulationen mit einem Schlage zu widerlegen. Diese allerdings grausame Methode hat zur Begründung einer wahren Physiologie mehr beigetragen als alle anderen Verfahren. «Haec crudelitas ad veram physiologiam plus contulit, quam omnes fere aliae artes.» ${ }^{112}$ Anatomische Beobachtungen und experimentelle Untersuchungen müssen in gleicher Anordnung und unter gleichen Bedingungen häufig wiederholt werden. Nur so können zufällige Störungen im Versuchsablauf (accidentes, aliena) erkannt und vom gültigen experimentellen Befund getrennt werden ${ }^{113}$. Physiologie ist Registrierung von Bewegungen (enarratio motuum) der Körpermaschine. Deshalb bedient man sich mit Recht der Mechanik und Hydraulik, um die Bewegungen und Kräfte zu beschreiben ${ }^{114}$. Aber hier ist umsichtige Kritik am Platze. Man muss die Komplexität und Eigenart der lebenden Maschine respektieren. Gewiss soll man die in der toten Natur waltenden Bewegungsgesetze auch auf den lebenden Organismus übertragen. Nur müssen dann die besonderen vitalen Bedingungen berücksichtigt werden. Die Blutströmung in elastischen und kontraktilen Gefäßen ist nicht identisch mit dem Wasserfluß durch starre Röhren ${ }^{115}$. Haller ist nicht nur qualitativ orientierter physiologischer Experimentator. Er versucht auch vitale Phänomene mathematisch zu beschreiben. Mikroskopische Untersuchungen bei Kaltblütern zeigten eine laminare Blutströmung mit maximaler Geschwindigkeit des Axialstroms in den kleinen Gefäßen. Dabei werden die Strömungsschichten verschiedener Geschwindigkeit durch die Blutkörperchen markiert (globuli frigidis certe in animalibus secundum lineas inter se et axi paralellas feruntur ${ }^{116}$. Der Strömungswiderstand in den Blutgefäßen ist proportional der Gefäßlänge sowie der inneren und äußeren Reibung des Blutes und umgekehrt proportional dem Querschnitt des Gefäßes ${ }^{117}$. Auch in dieser Studie kommt die empirische Orientierung Hallers zum Ausdruck: Zuerst die morphologische und physiologische Beobachtung und Messung, d.h., es wird die Komplexität der animalen Strukturen und Prozesse respektiert, dann folgt die darauf fußende Berechnung: Mathematik am Tier und nicht allein auf dem Papier. Bei diesen Herz- und Kreislaufstudien entfaltet Haller sein ganzes methodisches Rüstzeug: Morphologische Untersuchung, 
embryologische Studien, mikroskopische Beobachtung, experimentelle Analyse, physikalische Deutung, numerisch-statistische Auswertung der Befunde.

Als fundamentale Erscheinungen lebender Gebilde beschrieb Haller ihre Eigenschaften der Irritabilität und Sensibilität. Irritabel sind alle Gewebe, die sich auf mechanische, thermische oder chemische Stimuli zusammenziehen. Dieses Vermögen findet sich in ausgesprochener Weise beim Herzmuskel, den Skelettmuskeln und den Hohlmuskeln der Eingeweide ${ }^{118}$. Irritabilität ist nicht an das Nervensystem gebunden, auch isolierte Muskeln ohne Verbindung mit dem Rückenmark oder Gehirn zucken nach Reizung. Irritabilität kann nicht in ihrem Wesen erklärt werden, man kann sie nur empirisch registrieren (per experimenta invenitur). Auch die Irritabilität hat wie die Massenattraktion ihre Naturgesetzlichkeit (causa physica), die in der Beschaffenheit des Muskels gründet. Haller versucht nicht, diese «vis insita» oder "vis viva» auf bekannte physikalische Kräfte zu reduzieren. Das Konzept der Irritabilität ist aus den experimentellen Befunden und Beobachtungen (ex phaenomenis) streng empirisch gewonnen worden. Im Unterschied zur Gravitation blieb der Irritabilität eine mathematische Fassung versagt.

Die Elementa Physiologiae bilden eine monumentale Synthese des physiologischen Wissens bis zur Mitte des 18. Jahrhunderts. Neben einer Fülle von Fakten und einer straffen Gliederung des Stoffes verkörpern die Elementa die erste Darstellung der Probleme, Konzepte und Methoden der modernen Physiologie und bieten gleichzeitig das letzte Gesamtgemälde der machina humana als Ausklang der mechanistischen Iatrophysik.

\section{Anmerkungen}

1 Vgl. vor allem die Werke: De facultatibus naturalibus; De usu partium; De anatomicis administrationibus

2 s. Anm. 1, für die naturphilosophischen Wurzeln Galens vgl. insbes. De placitis Hippocratis et Platonis

3 Galen: De facultatibus naturalibus Lib. 1 cp. 12, Galen: On the natural faculties, Engl. transl. by A. J. Brock, London: W. Heinemann, Cambridge, Mass.: Harvard U. Pr. 1952, S. $42-49$

4 N. Mani: Die historischen Grundlagen der Leberforschung, Tl. 1: Die Vorstellungen über Anatomie, Physiologie und Pathologie der Leber in der Antike, Basler Veröffentl. z. Gesch.d. Med. u. Biologie, Fasc. IX, Basel 1959, S.61-77

5 Vgl. C. R.S.Harris: The heart and the vascular system in ancient Greek medicine, Oxford: Clarendon Pr. 1973, S. 349-365 
6 Galen: De placitis Hippocratis et Platonis, sec. pt., sec. ed., Lib. 7, cp.3, ed. Phillip de Lacy, Corpus Med. Graecor. V, 4, 1, 2, Berlin: Akad. Verl. 1984, S.438 (Ed. Kühn V 600)

7 S. Anm. 5 mit detaillierter Diskussion

8 Galen: In Hippocratis de natura hominis commentaria tria, ed. J. Mewaldt, Berlin/Leipzig: Teubner 1914, Corpus Medicor. Graecor. V, 9, 1, p.6; Galen: In Hippocratis librum de alimento commentarius, lib.3, cp.1 (Kühn XV, p. 252); Aristoteles: De generatione animalium, Engl.transl. A.L.Peck, London: Heinemann, Cambridge, Mass.: Harvard U.Pr. 1953, lib. 2, cp. 1, 4, 6, p. 152, 190, 212-214

9 S. Anm. 4 .

10 Galen: De anatomicis administrationibus, Lib. 8 cp. 5, 6 u. 9, Ed. Kühn II 677, 682-684, 696-698; Galen: On anatomical procedures, De anatomicis administrationibus, transl. by Ch.Singer, London/New York: The Wellcome Hist.Medical Museum, Oxford U.Pr. 1956, S.212, 214-215, 221-222; Galen: On Anatomical Procedures. The later books. Transl. by W.L.H.Duckworth, Ed. by M.C.Lyons, B.Towers, Cambridge U.Pr. 1962, Lib.IX, cp.13, S.20-24; Galen: De placitis Hippocratis et Platonis, Lib. 7 cp. 8, Kühn V 646; vgl. auch Anm. 6, S.476-477

11 Galen: De anatomicis administrationibus Lib. 8 cp. 9 (Kühn V 646); Galen: On anatomical procedures, the later books (s. Anm. 10) Lib.IX, cp.13-14, S.20-26; Galen: De placitis Hippocratis et Platonis Lib.2, cp.4 (Kühn V 239); Galen: On the doctrines of Hippocrates and Plato, Lib. 2 cp.4, ed. transl. by Ph. de Lacy, Pt. 1, Books I-V (Corpus Medicor. Graecor. V, 4, 1, 2 (1981) S.126-127

12 Galen: De usu partium, lib.7, cp.14, 15 (Kühn III 567-585), Lib.16, cp.4 (Kühn IV 278-289); Galen: On the usefulness of the parts of the body, transl. by M. T. May, Ithaca, N. Y.: Cornell U.Pr. 1968, p.368-371, p.688-694; Galen: De locis affectis, lib.1, cp.6 (Kühn VIII 52-57)

13 Galen: An in arteriis natura sanguis contineatur, cp. 2 (Kühn IV 707-708)

14 Galen: De facultatibus naturalibus, lib. 1 cp. 13 (Anm.3) S.58-59; Kühn II 36-37

15 Galen: Quod optimus medicus sit quoque philosophus, Kühn I 60-62; Galen: Methodus medendi, lib. VI cp. 4 (Kühn X 421)

16 Kühn I 59-62; Kühn X 310, 410

17 Kühn I 60; Kühn X 309, 359

18 Kühn X 310, 410

19 Kühn II 228, Kühn X 310

20 s. Anm. 11 Galen: On the doctrines of Hippocrates and Plato S. 78-80

21 Galen: De locis affectis, Lib.1, cp.6 (Kühn VIII 52-57)

22 Galen: De anatomicis administrationibus Lib. 7 cp. 12 (Kühn II $628 \mathrm{f}$.)

23 Galen: De anatomicis administrationibus Lib. 7 cp. 13, Lib. 8 cp. 3 (Kühn II 633, 664)

24 Benivieni, Antonio: De abditis nonnullis ac mirandis morborum et sanationum causis (lat. Orig. mit engl. Übers. durch Ch. Singer) Springfield: Thomans 1954

25 Esche, Sigrid: Leonardo da Vinci. Das anatomische Werk. Basel: Holbein Verl. 1954; Leonardo da Vinci on the human body. With transl. by Ch.D.O'Malley and J.B.C.M.Saunders, New York: Schuman 1952

26 Vesal, Andreas: De humani corporis fabrica, Basel 1543, 2. Aufl. Basel 1555

27 Adelmann, H.B.: The embryological treatises of H.Fabricius ab Aquapendente, Ithaca, N. Y.: Cornell U.Pr. 1942 
28 Coiter, Volcher: Diversorum animalium sceletorum explicationes iconibus artificiosis et genuinis illustratae, Nürnberg 1575, in: Opuscula selecta Neerlandicorum De arte medica, Bd. 18, Amsterdam 1955, S. 160-263

29 Mani, N.: Die Editio princeps des Galen und die anatomisch-physiologische Forschung im 16. Jahrhundert, in: Das Verhältnis der Humanisten zum Buch, hg. von F. Krafft und D.Wuttke, DFG, Kommission f.Humanistenforschung, Bonn: Deutsche Forschungsgemeinschaft 1977, S.209-226

30 Vesal, A.: De humani corporis fabrica (Anm.26) 1543, Lib. 7 cp. 19, S.658-663: De vivorum sectione nonnulla; Columbus, Realdus: De re anatomica, Venetiis 1559, Lib. 14, S. 256-261: De viva sectione

31 Vesal (Anm. 30) S.658

32 Colombo (Anm. 30) S. 256

33 Vesal (Anm.30) S.658-659 (eigentlich s.662-663); A.B.Baker: Artificial respiration, the history of an idea, in: Medical History 15 (1971) 336-351

34 Colombo (Anm. 30) De re anat., Lib.11, cp. 2, S.222-224

35 Mani, Nikolaus: Naturwissenschaftlich-biologische Grundlagenforschung in der Medizin des 17. Jahrhunderts, in: Medizinhistor. Journ. 11 (1976) S. 181-204

36 Ornstein, Martha: The rôle of scientific societies in the seventeenth century, Chicago: Un.Pr. 1928, S. 264-270

37 Gilbert, William: De magnete, magneticisque corporibus, et de magno magnete tellure; physiologia nova, plurimis et argumentis et experimentis demonstrata, London 1600; W. Gilbert: On the magnet, Ed. by D. J. Price, transl. S. P. Thompson, New York: Basic Books 1958; Balmer, Heinz: Beiträge zur Geschichte der Erkenntnis des Erdmagnetismus, Aarau: Sauerländer 1956 (Veröffentl.d.Schweiz.Ges.f.Gesch.d.Med. u.d. Naturwiss. 20) S. 149-163

38 Harvey, William: Exercitatio anatomica de motu cordis et sanquinis in animalibus, Frankfurt a.M. 1628, cp.8, S.41; cp.9, S.45; cp.10 S.46; cp.14 S.58; Pagel, Walter: William Harvey's biological ideas. Basel: Karger 1967

39 Stensen, Niels: De Glandulis oris et novis inde prodeuntibus salivae vasis, in: N. Steno: Opera philosophica, Ed. by V. Maar, Vol. 1, Copenhagen 1910, S. 15

40 Bartholin, Thomas: De lacteis thoracicis historia anatomica, Paris 1653, cp. 3 S.11: «Nos tantum credimus, quantum manibus palpamus oculisque videmus»

41 Wepfer, Johann Jacob: Observationes anatomicae ex cadaveribus eorum quos sustulit apoplexia, Schaffhausen 1658, S.36; Mähle, A.H.: Johann Jakob Wepfer als Toxikologe, Aarau: Sauerländer 1987 (Veröffentl.d.Schweiz.Ges.f.Gesch.d.Med.u.d. Naturwiss. 40)

42 Brunner, Johann Conrad: Experimenta nova circa pancreas. Amsterdam 1683, Epistola dedicatoria

43 Mani, N.: Die historischen Grundlagen der Leberforschung, Bd.2, Basel 1967, S. 104-120 (Basler Veröffentl. z. Gesch.d.Med. u. Biol.XXI)

44 Stensen, N.: De musculis et glandulis observationum specimen, Amsterdam 1664; Observationes anatomicae, quibus variae oris, oculorum et narium vasa describuntur, Leiden 1662

45 Malpighi, Marcello: De viscerum structura, Amsterdam 1669; M. Malpighi: Opere scelte a cura di Luigi Belloni, Torino 1967, S.153-187: sui reni 
46 Malpighi, Marcello: De pulmonibus observationes anatomicae, Bononiae 1661; id.: De pulmonibus epistola altera, Bononiae 1661

47 Van Leeuwenhoek, Antoni: Alle de brieven. The collected letters Vol.1-11, Amsterdam, später Lisse 1939-1983

48 Webster, Charles: The Helmontian George Thomson and William Harvey: The revival and application of splenectomy to physiological research, in: Medical History 15 (1971) S. 154-167

49 Brunner, Johann Conrad: Experimenta nova circa pancreas, Amsterdam 1683

50 De Graaf, Regnerus: Tractatus anatomico-medicus de succi pancreatici natura et usu, Leiden 1671

51 Buess, Heinrich: Die historischen Grundlagen der intravenösen Injektion, Aarau 1946 (Veröffentl.d.Schweiz.Ges.f.Gesch.d.Med.u.d.Naturwiss. XV); Isler, Hansruedi: Thomas Willis, ein Wegbereiter der modernen Medizin 1621-1675, Stuttgart 1965 (Grosse Naturforscher Bd.29) S. 14.1-144

52 Lower, Richard: Tractatus de corde, London 1669, S. 166-170

53 Swammerdam, Jan: Bibel der Natur, aus d.Holl. übers., Leipzig 1752, S.329-338: Versuche, die besondere Bewegung der Fleischstränge am Frosche betreffend

54 Gassendi, Pierre: Opera, T. 2, Lugduni 1658, S. 250-254: De anima cp. 3

55 Descartes, René: L'homme, et un traité de la formation du foetus, Paris 1664; Descartes über den Menschen (1632) sowie Beschreibung des menschlichen Körpers (1648), übers. von K. E. Rothschuh, Heidelberg 1969

56 Stensen, Niels: Myologiae specimen, seu musculi descriptio geometrica (Widmungsschreiben) in: N. Stensen: Opera Philosophica Vol.2, Copenhagen 1910, S.64; Stensen, N.: De glandulis oculorum, in: Stensen, N.: Opera Philos. Vol. I (1910) S. 81

57 Stensen, Niels: Epistolae, ed. G.Scherz, Vol. 2 Kopenhagen 1952, S. 921

58 Borelli, Giovanni Alfonso: De motu animalium, Hagae Comit., 1743 (Widmung an Königin Christine von Schweden 1679): «Tale, inquam, idioma et characteres, quibus Creator Rerum loquitur in suis operibus, sunt geometricae configurationes et demonstrationes»

59 Borelli, op.cit. z. B. Tabula XI, XV, XVI

59 a Borelli, op. cit., pars sec., cp.III, S.32-43

59 b Borelli, op. cit. S. 33

59c Bernoulli, Johann I.: De motu musculorum dissertatio physicomechanica, in: G. A. Borelli, De motu animalium, Hagae Comit. 1743

60 Stensen, Niels: Discours de Monsieur Stenon sur l'anatomie du cerveau, Paris 1669 (Fasc. Druck in:) Nicolaus Steno's Lecture on the anatomy of the brain, intr. by G. Scherz, Copenhagen 1965

61 Malpighi, Marcello: Risposta del Dottor Marcello Malpighi alla lettera intitolata: De recentiorum medicorum studio, in: Malpighi: Opere scelte a cura di Luigi Belloni, Torino 1967, S. 497-631

62 Malpighi, op. cit. S. 512

63 Malpighi, op. cit. S. 511-514

64. Malpighi, op. cit. S. 516

64 a Hooke, Robert: Micrographia, London 1665, The preface, p. (25) 
65 Santorio, Santorio: De medicina statica, Paris 1770 (erste der zahlreichen Auflagen Venedig 1614)

66 Galilei, Galileo: Opere, Edizione nazionale, Vol.12, Firenze 1902, Nr. 1080, S.140-142 Brief von Santorio an Galilei

67 Santorio, De med.statica. (Anm. 65) Aphor. IV, VI, S. 35-37

68 Van Helmont, Johannes Baptista: Sextuplex digestio § 13, 14 (Opera omnia, Frankfurt 1682, S. 201; idem: Calor efficienter non digerit § 26, 28 (Opera omnia (1682) S. 195; vgl. N. Mani (Anm.43) S.123-129

69 Sylvius, Franciscus Deleboe: Disputationes medicae, Amderdam 1663 VI § 46, S. 97; VII § 54, S. 119; Mani, N. (Anm. 43), S.129-131

70 Glisson, Francis: Tractatus de natura substantiae energetica, London 1672, Epistola dedicat., cp. VII § 10, S.90, cp. XIII § 6, S. 191 ff., S. 192: «Hoc igitur mihi probandum incumbit, naturam substantialem esse vivam, hoc est percipere, appetere et movere»

71 Stahl, Georg Ernst: Theoria medica vera, Halle 1708; Duchesneau, François: La physiologie des lumières. Empirisme, modèles et théories. Den Haag 1982; Gottlieb, B. J.: Bedeutung und Auswirkung des hallischen Professors und kgl. preuss. Leibarztes Georg Ernst Stahl auf den Vitalismus des XVIII. Jahrhunderts, Halle 1943, Nova Acta Leopoldina, N. F., Bd. 12 Nr. 89

72 Stahl, G.E.: Disquisitio de mechanismi et organismi diversitate, Halae 1706 (Dissertationes medicae, P.1, 4.)

73 s. M. Ornstein (Anm. 36); Purver, M.: The Royal Society, concept and creation, London 1967; Hahn, R.: The anatomy of a scientific institution: The Paris Academy of Sciences 1666-1803, Berkeley 1971; Winau, R.: Christian Mentzel und die Academia Naturae Curiosorum, Mainz 1970; vgl. dazu N. Mani (Anm. 35), S. 199-201

74 Malpighi (Anm. 61) S. 506; Stensen: Discours sur l'anat. du cerveau (Anm. 60)

75 Mani, N.: Ärzte und Naturforscher des 17.Jahrhunderts über die medizinische Forschung ihrer Zeit, in: Zusammenhang. Festschr.f. Marielene Putscher, hg. von O.Baur, O. Glandien, Bd. 1, Köln 1984, S. 353-365

76 Mani, N.: Jean Riolan and medical research, Bull. of Med. 42 (1968) S. 121-144

77 Swammerdam, J.: Bibel der Natur, Leipzig 1752, S. 338. Sp. 2; idem: Bybel der Natuure. Biblia naturae (hg. von) H. Boerhaave, (lat. Übers. von) H.D.Gaubius, T. II, Leyden 1738, S.835-860, (Facsimile-Ausgabe durch) B.V.Uitgeverij «de Banier»-Utrecht, Uitgeverij De Groot B. V.-Goodriaan 1980

78 Dewhurst, K.: Dr. Thomas Sydenham (1624-1689), his life and writings, Berkely, Los Angeles 1966, S. 63-65, 73, 85-93

79 Hales, Stephen: Statical essays containing haemastaticks, London 1733

80 Hales, Haemastaticks (Anm. 79) Preface, S. XII-XIII

81 Guerlac, Henry: Stephen Hales, in: Dictionary of Scientific Biography, Vol.VI, New York 1972, S.35-48; Circulation of the blood, men and ideas, ed. by A.P. Fishman, D.W. Richards, New York 1964, S.71-126: Output of the heart (W.F.Hamilton, D.W. Richards) St. Hales S.81-85, S.355-406: The capillary circulation by E.M.Landis, St. Hales S. 373-376

82 Hales, Haemastaticks (Anm. 79) Preface S. XIII

83 Hales op. cit. Dedication S. VI, VII, preface S. XIX (vgl. Weisheit Salomonis 11, 22)

84 Hales, op.cit. preface S. XIII-XV 
85 Hales, op.cit. preface, S. XVII, XVIII

86 Hales, op. cit., Introduction, Abschn. 4.5.

87 Hales, op. cit. S. 1-3

88 Hales, op. cit. S. 1-43 (42-43 Tabelle)

89 Hales, op.cit. S. 13-16, 42

90 op.cit. S. 55 (Experim. IX, 19.): «And to this resistance which the blood meets with in passing the capillary arteries, is owing the great difference of the force of the blood in the arteries to that in the veins, viz. as 10 or 12 to $1 . »$

91 Hales, op. cit. S.1-9

92 Hales, op.cit., preface S. XIX-XX; S. 32 (Experiment VII, 2.): «But the Allwise Framer of these admirable Machines has so ordered it, as that their healthy State shall not be disturbed by every little variation of this Force, but has made it consistent with a very considerable Latitude in the Variation of it.»

93 Hales, op. cit. S. 37-38 (Experiment VIII, 1.-6.)

94 Hales, op. cit. S. 39 (Experiment VIII, 8.)

95 Hales, op. cit. S. 22-23 (Experiment III, 26.)

96 Hales, op.cit. S. 48-63 (Experim. IX); S.126-131 (Experim. XV), S.131-133 (Experim. XVI); S. 133-143 (Experim. XVII); S. 135-139 (Experim. XVIII)

97 Hales, op. cit. S. 56 (Experim. IX 21.)

98 Bernoulli, Daniel: De vita. Eine akademische Festrede. Hg. von O.Spiess u. F. Verzar, in: Verhandlungen d.naturforschd.Ges. Basel, Bd.52 (1941) S.189-262; Bernoulli, Daniel: Recherches sur la manière la plus avantageuse de suppléer à l'action du vent sur les grands vaisseaux, in: Recueil des pièces qui ont remporté le prix de l'Académie Royale des sciences, Tome VII, Paris 1769 (prix de 1753); Huber, Friedrich: Daniel Bernoulli als Physiologe und Statistiker, Basel 1959, Basler Veröffentl.z.Gesch.d.Med.u.Biol. Fasc.VIII, S.56-61; Straub, Hans: Daniel Bernoulli, in: Dictionary of Scientific Biography Vol. II, New York 1970, S. 36-46

98 a Bernoulli, D.: Recherches (Anm. 98) S. 7-8

99 Hintzsche, E.: Albrecht von Haller, in: Dictionary of Scientific Biography, Vol. VI, New York 1972, S.61-67 (mit Bibliographie); Mani, Nikolaus: Albrecht von Haller und die Physiologie, in: Verhandlungen d.Schweiz. Naturforschd.Ges., Wiss.Teil, 1977, S.67-80 (mit Bibliographie); Toellner, R.: Albrecht von Haller. Über die Einheit im Denken des letzten Universalgelehrten, Wiesbaden 1971, Sudhoffs Archiv, Beihefte, H.10; Boschung, Urs: Albrecht von Hallers Aufenthalt in Paris im Lichte eines unbekannten Tagebuchs Johannes Gessners, in: Medizinhistor. Journ. 11 (1976) S. $220-245$

100 Haller, A.v.: Gedanken über Vernunft, Aberglauben und Unglauben, Vers 51-52, 55-56, in: Albrecht von Hallers Gedichte, hg. v. L. Hirzel, Frauenfeld 1882, S. 46

101 Newton, I.: Philosphiae Naturalis Principia Mathematica (3. ed., 1726), ed. by A. Koyré u.I.B.Cohen, Vol.2, Harvard U.Pr. 1972, S. 764 (ursprüngl.Pag. S. 530); Newton, I.: Opticks, New York: Dov.Publ.1952, Book 3 pt.1, S.401: «These principles (scl. gravity, fermentation, cohesion of bodies) I consider not as occult Qualities, supposed to result from specifick Forms of Things, but as general Laws of Nature, by which the Things themselves are form'd; their Truth appearing to us by Phaenomena, though their Causes be not yet discover'd.» 
102 Haller, Elementa Physiol. T.IV, S.531; Elmenta Physiol.I, S.426; Vgl. dazu: Töllner (Anm.99) S. 144-146: Duchesneau (Anm. 71) S.148-156; Rudolph, G.: Hallers Lehre von der Irritabilität und Sensibilität, in: Von Boerhaave bis Berger. Die Entwicklung der kontinentalen Physiologie im 18. und 19. Jahrhundert, hg. von K. E. Rothschuh, Stuttgart 1964, S. 14-34 (S.27-29)

103 Elementa Physiol. I, S. 426

103 a Haller, Elementa Physiol. T. I, p.I (praef.)

104 Haller: Primae lineae physiologiae, Göttingen 1747 (praef.); Elementa Physiol.I, S. I-III

105 Haller: Elementa Physiol. I, S. I-II

106 Haller: De amoenitate anatomes, in: Opera Minora III, S. 272-276

107 Haller: Elementa Physiol. I S. III

108 Haller: Elementa Physiol. I S.IV

109 Haller: Elementa Physiol. IV, S. 458

110 Haller: Elementa Physiol.IV, S. 316-319; V S.538-539

111 Haller: Elementa Physiol. I S. III-IV

112 ibid.

113 Elementa Physiol.I S. V; Haller: Mémoires sur la nature sensible et irritable des parties du corps animal, Lausanne 1756, S.110, 114-115

114. Haller: Elementa Physiol. I S. V-VI

115 ibid. S.VI

116 Haller: Elementa Physiol. II S. 323

117 ibid. S.178-184, 272-282; Wobmann, P.: Albrecht von Haller, der Begründer der modernen Haemodynamik, in: Arch.f.Kreislaufforschung Bd.52 (1967) S.96-128 (S.177f.)

118 Haller: De partibus corporis humani sensilibus et irritabilibus, in: Commentarii Soc. Reg. Scientiarum Gottingensis Bd.2, Göttingen 1753, S.114-158 (S.154); Elementa Physiol.IV, S.464, 514

\section{Summary}

\section{Physiological concepts from Galen to Haller}

Galenic physiology is based on Platonic natural philosophy, Aristotelian biology and teleology and on Alexandrian anatomical research and physiological and medical experiments. Most remarkable is Galen's strong anatomical and structural orientation of research and his unusual experimental analysis of physiological functions although the results of physiological experiments are not the main source and ultimate criterion of physiological knowledge but rather confirm the overall theoretical framework of Galen's medical and biological thought.

In the seventeenth century a strong empirical trend pervades physiological research. Anatomical dissection, physiological experimentation, comparative anatomy, biological reasoning and quantitative thought are the roots of Harvey's discovery of the blood circulation. The scientific revolution of this epoch leads to mechanistic explanations of 
physiological phenomena (Descartes) and to a geometrical demonstration and mathematical calculation of vital processes (Borelli, Bernoulli). Stephen Hales' "haemastaticks" blends most successfully Newtonian thought, ingenious animal experimentation and elementary mathematical calculation in order to assess the physical laws and the biological conditions of the blood flow. Chemical views as fermentation (van Helmont) and the effervescence between alkaline and acid substances (Sylvius de le Boe) endeavor to explain physiological and pathological phenomena. Besides, hylozoistic and vitalistic concepts (Glisson) and animistic interpretation of vital properties (Stahl) try to understand the forces governing the living body, f.i. the concept of irritability (Glisson) as basic property of living matter to respond to various internal and external stimuli.

Albrecht von Haller's Elementa Physiologiae form a monumental synthesis of physiological knowledge and present the first modern view of physiology as a coherent and independent discipline. Haller defines the problems, methods and concepts of physiology. Physiology describes the vital motions and chemical changes of the animal machine as moving anatomical structures. The key method is experimentation on the living animal. Haller also endeavors to calculate the physical phenomenon of blood flow while always respecting the complex conditions of living systems.

Prof. Dr. med. Nikolaus Mani

Medizinhistorisches Institut

Sigmund-Freud-Str. 25

D-5300 Bonn 1 